Prevention of stroke

\section{Should I start all my ischaemic stroke and TIA patients on a statin, an ACE inhibitor, a diuretic, and aspirin today?}

\section{P A G Sandercock}

$\mathrm{S}$ troke is eminently preventable. ${ }^{12} \mathrm{~A}$ combination of individual and population based interventions could lower the global incidence of vascular events by as much as $50 \%{ }^{2}$ However, the public health and legislative changes required to achieve substantial primary prevention of vascular disease (for example, by reducing the salt content of processed food) is really the territory of public health systems and governments ${ }^{2}$ and is beyond the scope of this article. In this short review I shall therefore deal with stroke prevention from the perspective of the hospital clinician and hence focus on secondary prevention. In the 1980s, the only treatment offered to many patients with transient ischaemic attack (TIA) or minor ischaemic strokes was aspirin. There is now a much wider range of evidence based interventions available for reducing the risk of recurrent stroke and other serious vascular events. ${ }^{1}$ In brief, I will identify who is at high enough risk to justify intervention; which interventions are effective; and how best to arrange neurovascular services to deliver the interventions efficiently.

\section{WHO IS AT HIGHEST RISK? \\ Individuals at high absolute risk of vascular events}

In neurological and neurosurgical practice, the people who have most to gain from secondary prevention are those with minimal or no disability who are at highest absolute risk of disabling stroke-that is, those who have had a recent TIA or minor stroke. Some individuals with atrial fibrillation but no history of a cerebrovascular event may have a comparably high absolute risk. If one follows up such individuals, they are likely to suffer not just strokes but also myocardial infarcts, or to require vascular surgical procedures (on the cerebral, coronary or peripheral arteries), or to die from vascular causes. For an intervention such as carotid endarterectomy for symptomatic carotid stenosis (with an average 3-5\% risk of fatal or disabling stroke complicating the procedure), it is important to offer it only to those individuals at sufficiently high absolute risk of stroke to justify the hazard. On the other hand, in patients aged under 65 with non-rheumatic atrial fibrillation, no history of stroke or TIA and no vascular risk factors (that is, "lone atrial fibrillation"), the annual risk of stroke is well below $2 \%$, and aspirin (rather than warfarin) becomes the antithrombotic agent of choice. ${ }^{3}$ To aid the process of clinical decision making there are predictive models that may help identify patients at high and low risk of vascular event after stroke or TIA, ${ }^{4}$ and risk stratification models for patients in atrial fibrillation. $^{3}$

\section{Patients with recent events}

When a patient is referred to hospital with a TIA or a stroke, this is an opportunity to prevent further disabling vascular events. The risk of stroke is at its highest in the first few days after the event (and is higher than was previously estimated). ${ }^{5}{ }^{6}$ Patients must therefore be seen, investigated, and started on effective treatment as promptly as possible (within a few days) before a disabling stroke occurs. In the United Kingdom, the national guideline stipulates that patients with TIA and minor stroke not immediately admitted to hospital should be assessed at a neurovascular clinic within 14 days of symptom onset (but even this may be too late for some).

Even a remote past history of TIA or stroke is important

However, the increased risk persists, in those that survive the high risk early period. ${ }^{8}$ Preventive treatments should therefore be considered even in people with a past history of TIA or ischaemic stroke several years before, and so there is a case for treatment to be continued indefinitely (though one must appreciate the limitation that many of the prevention trials tested treatment periods of only two to five years).

\section{HOW CAN WE ASSESS AND INVESTIGATE HIGH RISK PATIENTS EFFICIENTLY? Comprehensive service to reach all high risk patients}

The first problem is to ensure that all patients who might benefit from secondary prevention strategies are identified, assessed systematically, and given appropriate treatment promptly. This is not as easy as it seems, as patients may present to outpatients, to the emergency department, or be admitted to a medical, neurological, neurosurgical, or other ward. "Fast track TIA clinics" are very helpful because they can arrange the speedy and efficient assessment of patients suspected of having acute cerebrovascular disease that is not severe enough to require hospital admission. ${ }^{7}$ The first priority is to weed out patients who have migraine, focal epilepsy, syncope, brain tumours, and other non-vascular problems as the cause for their transient focal neurological disturbances. It is now even more important to distinguish transient focal neurological symptoms with a vascular cause from those with a non-vascular cause, as the former may require a lifelong prescription of up to four drugs (see below) and the latter none. For patients who have had focal symptoms lasting more than a few hours, computed tomography or magnetic resonance imaging to identify the pathological type of underlying lesion (ischaemic, haemorrhagic, or non-vascular) is an essential first step. ${ }^{7}$ Patients with carotid territory TIA or non-disabling ischaemic stroke should be fast tracked for non-invasive ultrasound carotid artery imaging, and those found to have severe symptomatic stenosis should have carotid endarterectomy within one or two weeks, before disabling stroke occurs. The choice of imaging tests to confirm the stenosis is a matter for debate; many clinicians

Abbreviations: ASCOT, Anglo-Scandinavian cardiac outcomes trial; AVASIS, aspirin versus anticoagulants in symptomatic intracranial stenosis; ESPRIT, European/Australian stroke prevention in reversible ischaemia trial; GALA, general anaesthetic versus local anaesthetic for carotid endarterectomy; PROGRESS, perindopril protection against recurrent stroke study; PROSPER, pravastatin in elderly individuals at risk of vascular disease; SPORTIF, stroke prevention by oral thrombin inhibition IV; TIA, transient ischaemic attack; WARCEF, warfarin-aspirin reduced cardiac ejection fraction study; WASID, warfarin-aspirin symptomatic intracranial disease study 
now use ultrasound plus one other noninvasive test for confirmation, whereas others regard intra-arterial arteriography as the best final arbiter of surgical decisions, despite the risks. ${ }^{9}$ Finally, one should not neglect patients with TIAs and minor strokes in the vertebrobasilar territory, as they have recently been shown to have a prognosis at least as bad as, if not worse than, carotid territory events, ${ }^{10}$ and should now merit an equally aggressive approach to secondary prevention (outlined below).

\section{The benefits of a systematic approach}

It may go against the grain of clinical freedom, but checklists and proformas can increase the chance that the clinician identifies all the many key data items relevant to management: causative factors for the stroke; modifiable risk factors; important social data (for example, smoking habit, whether or not the patient drives a car); their occupation; and, most importantly, the patient's knowledge of stroke and its risk factors.

\section{RISK FACTOR MANAGEMENT FOR ALL PATIENTS}

\section{Blood pressure reduction}

In observational studies, the relation between blood pressure, stroke, and vascular mortality is now characterised as log-linear, the risk of stroke and vascular mortality falling the lower the blood pressure, with no evidence of a threshold down to $115 / 75 \mathrm{~mm} \mathrm{Hg}^{11}$-in other words, the lower, the better. The PROGRESS trial showed that blood pressure reduction with a combination of an angiotensin converting enzyme (ACE) inhibitor and a diuretic benefited all types of patients with a recent cerebrovascular event, irrespective of their baseline blood pressure. ${ }^{12}$ The benefits were evident whether the drugs were taken alone or in addition to existing antihypertensive treatment. ${ }^{12}$ Blood pressure reduction, importantly, lessened the recurrent risk of stroke after both ischaemic and haemorrhagic stroke. ${ }^{12}$ The benefits were consistent with those in other trials of ACE inhibitors. ${ }^{12}{ }^{13}$ The specific agents tested in the PROGRESS trial were indapamide and perindopril, but there is reasonable evidence that other diuretic and ACE inhibitor regimens, and blood pressure reductions with other agents, are likely to be effective as well. ${ }^{13}$ For patients who cannot tolerate ACE inhibitors (for example, because of cough), blood pressure reduction with other agents (such as calcium antagonists or $\beta$ blockers) is likely to reduce the risk of stroke, though the evidence of benefit comes more from studies in primary stroke prevention.

\section{Smoking cessation}

Smoking is a risk factor for all forms of vascular disease, and there is reasonable evidence that avoidance of smoking is likely to reduce the risk of further vascular events. ${ }^{14}$ Smoking cessation is undoubtedly helped by nicotine replacement therapy in the form of patches or gum. ${ }^{14}$

\section{INTERVENTIONS FOR PATIENTS WITH ISCHAEMIC EVENTS Cholesterol reduction with statins}

The MRC/BHF heart protection study reliably showed that, among patients with a history of previous coronary heart disease, stroke or TIA, or diabetes, simvastatin $40 \mathrm{mg}$ daily not only reduced the risk of stroke, myocardial infarction, and death from vascular causes, but also the need for vascular surgical procedures (on the coronary and carotid arteries), by about a quarter. ${ }^{15}$ This trial showed that statins benefit high risk individuals both with and without a history of previous coronary heart disease, as well as those with a total cholesterol in the range 3.5 to $5.0 \mathrm{mmol} / \mathrm{l}$, and that the benefits of cholesterol reduction depend chiefly on the individual's overall risk of major vascular events, rather than on their blood lipid concentrations alone. ${ }^{15}$ Two recently published trials-PROSPER and ASCOT-add weight to this evidence of benefit from cholesterol reduction in older people $\mathrm{e}^{15-17}$ and in reducing the risk of stroke. ${ }^{17}$ These trials have robustly confirmed the safety of statins, dismissing earlier concerns about increases in non-vascular deaths ${ }^{15} 16$ (chiefly certain site specific cancers and suicide), and thus reinforcing a recent American College of Cardiology/ American Heart Association/National Heart Lung and Blood Institute advisory highlighting underuse of statins because of unfounded safety concerns about muscle and liver toxicity. ${ }^{18}$

\section{Antiplatelet treatment}

There is very strong evidence that long term antiplatelet treatment reduces the risk of serious vascular events after TIA and minor stroke, and the greatest body of evidence exists for aspirin at a dose of 75 to $150 \mathrm{mg} /$ day. ${ }^{19}$ For patients genuinely intolerant of aspirin, clopidogrel $75 \mathrm{mg} /$ day is an alternative. ${ }^{19} 20$ One large trial has suggested that the combination of modified release dipyridamole and aspirin is more effective than aspirin alone, but systematic reviews suggest the extra benefit may be only modest (or even negligible). ${ }^{19-25}$ Trials are under way to assess which of the many combinations of antiplatelet drugs is best (aspirin $v$ aspirin plus clopidogrel, clopidogrel $v$ clopidogrel plus aspirin, aspirin plus clopidogrel $v$ dipyridamole plus aspirin).*

\section{Anticoagulants for patients in atrial fibrillation}

Among patients with atrial fibrillation and an ischaemic event in the brain, provided there are no contraindications (such as probable poor compliance with anticoagulants, a history of falls or recent gastrointestinal bleeding), oral anticoagulants substantially reduce the risk of stroke. ${ }^{22}$ The target international normalised ratio (INR) should be 2.5, with a range from 2.0 to 3.0. The challenge is to deliver this labour intensive intervention to all those most likely to benefit. Achieving appropriate screening, risk stratification, and delivery of oral anticoagulants for primary stroke prevention in atrial fibrillation is an even greater challenge. ${ }^{3}$ The SPORTIF-III trial, recently reported but not yet published in full, suggested that ximelagatran, an oral direct thrombin inhibitor, may be no less effective than warfarin for primary prevention of vascular events in atrial fibrillation but is more convenient; a second phase III randomised trial (SPORTIF-V) is still under way. ${ }^{23}$ The results have some relevance to secondary prevention, as about a quarter (perhaps 800 or so) of the 3407 patients in SPORTIF-III had a history of previous stroke or TIA..$^{23}$ Full publication of both trials will be needed to assess the place of this new agent in secondary stroke prevention.

\section{Anticoagulants for patients in sinus rhythm}

For patients in sinus rhythm, there is no evidence that anticoagulants offer any clear net benefit (when compared with either control ${ }^{24}$ or antiplatelet agents ${ }^{25}$ ). There are several trials under way comparing various warfarin regimens with antiplatelet agents in different categories of patient without atrial fibrillation (ESPRIT, WASID, AVASIS, WARCEF) (see footnote)

${ }^{*}$ An up to date list of ongoing and recently completed trials of interventions for secondary stroke prevention can be found at the Internet Stroke Center at Washington University (http:// www. strokecenter.org/trials/browse $/$ asp? browse $=$ prevent\&condition $=4) \quad($ date last accessed 18 April 2003). 
Surgery, angioplasty, or stents for symptomatic carotid stenosis

For patients with recently symptomatic severe stenosis of the internal carotid artery $(>70 \%$ stenosis by the NASCET method), there is strong evidence that carotid endarterectomy is highly beneficial; the balance of risk and benefit is less clear for moderate stenosis. ${ }^{26}$ There is debate about the cost-effectiveness of the procedure. ${ }^{27}$ Patients must of course accept that surgery carries a small but definite risk of disabling stroke or death, and the surgeon performing the procedure should have an independently audited major complication rate of $5 \%$ or less. The local surgical service should aim to perform surgery as soon as possible (surgery much later than a month or two after the TIA is largely futile as the high risk period without surgery will have passed). The GALA trial seeks to determine whether surgery is best done under local or general anaesthesia. ${ }^{28}$ Primary angioplasty of the carotid artery with or without stenting holds the promise of being simpler, less invasive, and as effective as endarterectomy. ${ }^{29}{ }^{30}$ Various small trials comparing surgery with angioplasty with or without primary stenting have been completed and others are under way (see footnote). For the time being, there is no reliable large scale trial evidence to guide the choice of alternatives to carotid endarterectomy.

\section{CONCLUSIONS}

There are many obstacles to be overcome if we are to achieve comprehensive and effective services for patients at high risk of disabling stroke. The first is to educate the general public on the symptoms of stroke and transient ischaemia. The second is to ensure that when symptoms occur, effective prevention is started immediately, before disabling stroke occurs. Fast track outpatient clinics offering a comprehensive "one stop" clinical and investigational service can make secondary preventive care more effective. The need for accurate diagnosis, the wide range of specialist investigations, and the types of treatments that can now be offered are such that the early management should generally be provided by specialists in secondary care and not by primary care physicians. Neuroradiology and vascular surgical services must be streamlined to ensure that appropriately selected patients are treated within the evidence based time limits now enshrined in clinical standards. Finally, to return to the question posed in the title: if a 70 year old man is referred to the clinic with a single episode of transient weakness of the hand and arm, and after appropriate assessment is judged to have had a transient ischaemic attack, should he be sent away on aspirin, a statin, an ACE inhibitor, and a diuretic? The answer is "generally, yes." And the exceptions to this general rule? I have tried to summarise some of my own personal exceptions (table 1); other clinicians will undoubtedly formulate their own.

\section{ACKNOWLEDGEMENTS}

I would like to thank the following for their helpful comments on drafts of the manuscript: R Davenport, R Hart, M Dennis, J Wardlaw, C Sudlow, C Warlow, P Rothwell, R Al-Shahi, R Lindley, D Leys, P Enevoldson.

\section{J Neurol Neurosurg Psychiatry}

2003;74:1461-1464

\section{Author's affiliation \\ P A G Sandercock, Department of Clinical Neurosciences, Western General Hospital,} Edinburgh EH4 2XU, UK

Competing interests: The author is co-principal investigator for the third International Stroke Trial (IST3) of thrombolysis in acute stroke. The trial is funded by grants from the Stroke Association and PPP Healthcare Trust.

Boehringer Ingelheim have donated trial drug and placebo. The author has received honoraria for lectures, travel expenses, and ad hoc consultations from a variety of pharmaceutical companies including Boehringer Ingelheim, Sanofi, BMS, MSD, Servier, Glaxo-Wellcome, Lilly, Centocor, and Bayer.

Table 1 A set of (my personal) exceptions to the rule that all patients with definite transient ischaemic attack or ischaemic stroke should be treated with aspirin, a statin, an ACE inhibitor, and a diuretic

General exceptions

- There is doubt that the symptoms were of vascular origin

- The patient is at very low risk of vascular events (for example, a single episode of amaurosis fugax in a patient with no evidence of carotid atheroma and no evidence of coronary heart disease or periphera vascular disease)

Exceptions for specific drugs (apart from their usual contraindications)

- Aspirin: clear history of aspirin allergy; recent gastrointestinal bleeding

- Statin: total cholesterol $<3.5 \mathrm{mmol} / \mathrm{l}$

- ACE inhibitor/diuretic/blood pressure lowering and (a) blood pressure $<115 / 75 \mathrm{~mm} \mathrm{Hg}$; (b) severe occlusive arterial disease in the neck (for example, bilateral carotid occlusion)

- The patient is already on a very large number of drugs

This list is not comprehensive and aims to give examples to illustrate the type of exceptions to consider.
Correspondence to: Professor Sandercock; pags@skull.dcn.ed.ac.uk

\section{REFERENCES}

1 Straus S, Majumdar SR, McAlister FA. New Evidence for stroke prevention. Scientific review. JAMA 2002;288:1388-95

2 Murray CJL, Lauer JA, Hutubessy RCW, et al Effectiveness and costs of interventions to lower systolic blood pressure: a global and regional analysis on reduction of cardiovascular disease risk. Lancet 2003;361:717-25.

3 Wang TJ, Massaro JM, Levy D, et al. A risk score for predicting stroke or death in individuals with new onset atrial fibrillation in the community. The Framingham Heart Study. JAMA 2003;290: 1049-56.

4 Rothwell P, Warlow C. On behalf of the European Carotid Surgery Trials' Collaborative Group. Lancet 1999:353:2105-10, On line version available at http://www.dcn.ed.ac.uk/ model/models.asp (last accessed 18 April 2003).

5 Johnston SC, Gress DR, Browner WS, et al. Shortterm prognosis after emergency department diagnosis of TIA. JAMA 2000;284:2901-6.

6 Lovett JK, Dennis MS, Sandercock P, et al. Very early risk of stroke after a first transient ischemic attack. Stroke (Online) 2003;32:e138-42.

7 Intercollegiate Working Party for Stroke (Update 2002). London: Royal College of Physicians, 2002; (available at http://www.rcplondon.ac.uk/ pubs/books/stroke/StrokeUpdate2002.pdf).

8 Clark TG, Murphy MFG, Rothwell P. Long term risks of stroke, myocardial infarction and vascular death in "low risk" patients with a non-recent transient ischaemic attack. J Neurol Neurosurg Psychiatry 2003;74:577-80.

9 Warlow CP, Dennis MS, van Gijn GJ, et al. Stroke. A practical guide to management, 2nd ed. Oxford: Blackwell Scientific Publications, 2000:271-2.

10 Flossmann E, Rothwell PM. Prognosis of vertebrobasilar transient ischaemic attack and stroke. Brain 2003;126:1940-54.

11 Prospective studies collaboration. Age-specific relevance of usual blood pressure to vascular mortality: a meta-analysis of individual data for one million adults in 61 prospective studies. Lancet 2002;360:1903-13.

12 PROGRESS Collaborative Group. Randomised trial of a perindopril-based blood-pressurelowering regimen among 6105 individuals with previous stroke or transient ischaemic attack. Lancet 2001;358:1033-41.

13 Bath P. Blood-pressure lowering for secondary prevention of stroke: ACE inhibition is not the key Stroke. 2003;34:1334-5.

14 Warlow CP, Dennis MS, van Gijn GJ, et al. Stroke. A practical guide to management, 2 nd ed. Oxford: Blackwell Scientific Publications, 2000:672.

15 Heart Protection Study Collaborative Group. MRC/BHF heart protection study of cholestero lowering with simvastatin in 20536 high-risk individuals: a randomised placebo-controlled trial. Lancet 2002;360:7-22.

16 Shepherd JI, Blauw GJ, Murphy MB, et al. Pravastatin in elderly individuals at risk of vascular disease (PROSPER): a randomised controlled trial. Lancet 2002;360:1623-30.

17 Sever PS, Dahlof B, Poulter N, et al. Prevention of coronary and stroke events with atorvastatin in hypertensive patients who have average or lowerthan average cholesterol concentrations, in the Anglo-Scandinavian cardiac outcomes trial-lipid lowering arm (ASCOT-LLA): a multi-centre randomised controlled trial. Lancet 2003:361:1149-58.

18 Pasternak RC, Smith SC, Bairey-Merz CN, et al. ACC/AHA/NHLBI clinical advisory on the safety of statins. Stroke 2002;33:2337-41.

19 Antithrombotic Trialists' Collaboration Collaborative meta-analysis of randomised trials of antiplatelet therapy for the prevention of death, myocardial infarction, and stroke in high risk patients. BMJ 2002;324:71-86

20 Hankey GJ, Sudlow CLM, Dunbabin DW Thienopyridines or aspirin to prevent stroke and 
other serious vascular events in patients at high risk of vascular disease? A systematic review of the evidence from randomized trials. Stroke 2000:31:1779-84

21 De Schryver ELLM, Algra A, van Gijn J. Dipyridamole for preventing stroke and other vascular events in patients with vascular disease (Cochrane review). In: The Cochrane Library, Issue 1. Oxford: Update Software, 2003.

22 Hart RG, Benavente O, McBride R, et al. Antithrombotic therapy to prevent stroke in patients with atrial fibrillation: a meta-analysis. Ann Intern Med 1999;131:492-501.

23 Halperin J. on behalf of the SPORTIF-III investigators. Presented at 52nd meeting of the American College of Cardiology. http:// www.acc03online.org/ondemand/trials3/ sessions. asp? sid $=3$.
24 Sandercock P, Mielke O, Liu M, et al. Anticoagulants for preventing recurrence following presumed non-cardioembolic ischaemic stroke or transient ischaemic attack (Cochrane Review). In: The Cochrane Library, Issue 1. Oxford: Update Software 2003.

25 Algra A, De Schryver ELLM, van Gijn J, et al. Oral anticoagulants versus antiplatelet therapy for preventing further vascular events after transient ischaemic attack or minor stroke of presumed arterial origin (Cochrane Review). In: The Cochrane Library, Issue 1. Oxford: Update Software, 2003

26 Rothwell PM, Eliasziw M, Gutnikov SA, et al. For the Carotid Endarterectomy Trialists'

Collaboration. Analysis of pooled data from the randomised controlled trials of endarterectomy for symptomatic carotid stenosis. Lancet 2003;361:107-16.

27 Benade M, Warlow CP. Costs and benefits of carotid endarterectomy and associated preoperative arterial imaging. A systematic review of the health economic literature. Stroke 2002;33:629-38

28 The GALA trial collaborative group. The GALA trial. http://www.den.ed.ac.uk/GALA.

29 Crawley F, Brown MM. Percutaneous transluminal angioplasty and stenting for carotid artery stenosis (Cochrane Review). In: The Cochrane Library, Issue 1. Oxford: Update Software, 2003.

30 CAVATAS Investigators. Endovascular versus surgical treatment in patients with carotid stenosis in the carotid and vertebral artery transluminal angioplasty study (CAVATAS): a randomised trial. Lancet 2001;357:1722-3.

\section{Psychopathology in people with epilepsy and intellectual disability}

\section{F M C Besag}

\section{Both behavioural and psychiatric disorders are common in people with learning disability and epilepsy}

$\mathrm{F}$ rom the time of the Isle of Wight study ${ }^{1}$ it became evident that children with epilepsy who also had other brain problems had a high rate of behavioural disturbance. Although there is a lack of good epidemiological studies of behavioural/psychiatric disorders in adults with epilepsy and learning disability (intellectual disability, mental retardation), it appears that such disorders are common. ${ }^{2}{ }^{3}$ Any serious attempt to determine the factors responsible for these associations is to be welcomed. The paper by Espie et al on p $000^{4}$ is in this category and the authors are to be congratulated on raising some important issues. Their results reflect, to some extent, the findings of earlier studies on people with learning disability, notably in the papers by Deb and Hunter, ${ }^{2}$ that neither behavioural nor psychiatric disorders are more common in those with epilepsy. However, Espie et al conclude that some epilepsy specific factors may be associated with psychiatric disorder.

When the aim of a study is to determine predictive factors for behavioural/psychiatric disorders by comparing populations, it is very important to ensure that the results are not confounded by selection bias in either the study group or comparison groups. It is also important to use measures that are valid and reliable. The statistical methods should be appropriate and overemphasis should not be placed on factors associated with only a relatively small proportion of the variance. Finally, there has been a tendency in publications in this field either to be anecdotal, with great relevance to everyday practice but with a weak scientific basis, or to present rather dry multiple statistical analyses ("statistical fishing trips") that seem to bear little relation to real life.

In the study by Espie et al the authors declare the shortcomings of both the study and comparison groups. As a measure of psychiatric disorder, they use the PAS-ADD checklist, which is primarily intended as a screening schedule to indicate whether further mental health assessment may be required, rather than the full PAS-ADD (psychiatric assessment schedule for adults with developmental disability) or the mini PAS-ADD.

Espie et al have raised another matter which is too often ignored, namely the profound impact that caring for someone with learning disability can have on the carers. This aspect of their paper is particularly worthwhile.

The overall conclusions remain that both behavioural and psychiatric disorders, whatever reasonable definitions are used, are common in people with learning disability and epilepsy but that it is probably largely factors other than the epilepsy itself that are responsible for this high prevalence. The learning disability is of major relevance. Other factors that should be considered include sensory impairments and communication difficulties. Adverse drug effects and epilepsy related factors are probably causal in a relatively small pro portion of this population but they are very important because they can often be rectified by correct management. ${ }^{5}$

There is still a great need for carefully designed and meticulously conducted large scale epidemiological studies of behavioural and psychiatric disturbance in people with learning disability and epilepsy. Such studies should inform us in our endeavours to reduce morbidity both in these individuals and in their carers.

J Neurol Neurosurg Psychiatry 2003;74:1464

\section{Author's affiliation}

F M C Besag, Bedfordshire and Luton Community NHS Trust, Bedford MK41 6AT, UK and Centre for Epilepsy, The Maudsley Hospital, London, UK

Correspondence to: Professor F M C Besag; FBesag@aol.com

\section{REFERENCES}

1 Rutter M, Graham P, Yule W. A neuropsychiatric study in childhood. London: Heinemann Medical, 1970

2 Deb S, Hunter D. Psychopathology of people with mental handicap and epilepsy. I. Maladaptive behaviour. Br J Psychiatry 1991;159:822-6.

3 Deb S, Hunter D. Psychopathology of people with mental handicap and epilepsy. II. Psychiatric illness. Br J Psychiatry 1991;159:826-30.

4 Espie CA, et al. Psychopathology in people with epilepsy and intellectual disability; an investigation of potential explanatory variables. J Neurol Neurosurg Psychiatry 2003;74: 1485-92.

5 Besag FMC. Behavioural effects of the new anticonvulsants. Drug Safety $2001 ; 24: 513-36$. 
Neuroplasticity and rehabilitation

\section{Stroke: neuroplasticity and recent approaches to rehabilitation}

\section{R M Bracewell}

$\mathrm{t}$ is good to see that stroke, and in particular stroke rehabilitation, is losing its Cinderella status in the world of neurology. In this issue, Cauraugh and Kim ( $p$ 000) present the results of some recent work on the rehabilitation of arm function in chronic stroke patients. ${ }^{1}$ This work touches on several areas of interest to both clinicians and basic scientists.

Their work was in part motivated by suggestions in the motor learning literature that the repetitive practice of the same action ("blocked" practice) may be inferior to practising different actions ("random" practice). If this were so, then it would have important implications for everyday clinical practice, in which patients are typically required to perform the same action time and again (the equivalent of blocked practice). With the particular training schemes used, however, no difference was found between the two groups.

A more positive result came from a comparison of the performance of patients allocated to receive or not neuromuscular stimulation assistance. In the assisted group, once the EMG level in the voluntarily activated muscle exceeded a threshold value, electrical stimulation was delivered to that muscle to assist the action. Patients who received such assistance fared better than those who received traditional physical therapy. Others have reported similar beneficial effects of such assistance in stroke and paraplegia. We have used an active computer joystick to guide the responses of chronic stroke patients in a novel approach to cognitive rehabilitation. ${ }^{2}$ It is likely that the use of such technologies will play an increasingly important role in stroke rehabilitation.

The authors also showed that the impaired arm tended to perform better when tested during bimanual actions than when used alone. We know, of course, that many everyday actions are bimanual, and that there is extensive neural machinery involved in the coordination of bimanual action. Rather than focusing on the retraining of the impaired arm alone, perhaps we should be adopting a more even handed-and cheap-approach to the rehabilitation of hemiparesis.

Following stroke in man, there is evidence of plastic reorganisation in both hemispheres. A consensus view is emerging that plasticity in the hemisphere contralateral to the affected limbs is more important for recovery than reorganisation on the other side. ${ }^{3}$ Indeed, more complete recovery appears to be associated with a more normalthat is, relatively restricted and contralateral-pattern of brain activation. ${ }^{4}$ Nudo et al have shown in non-human primates that the primary motor cortex (M1) can reorganise after a focal vascular lesion if there is motor skill retraining. In animals not trained after a stroke, there is a further reduction in the size of the hand representation in M1. ${ }^{5}$ Cauraugh and Kim showed that neuromuscular stimulation assistance improved the ability of chronic stroke patients to use their paretic arms. One might speculate that this assistance increases the likelihood of appropriate plasticity in the motor areas, particularly on the contralateral side. This suggestion could be pursued with transcranial magnetic stimulation and functional imaging studies.

These are exciting developments. Findings from animal neurophysiology, human behavioural neuroscience and imaging studies, and the use of technological assistance hold out the prospect of more rational and effective rehabilitation strategies for the victims of stroke.

J Neurol Neurosurg Psychiatry 2003;74:1465

\section{Author's affiliation}

R M Bracewell, Centre for Cognitive Neuroscience, School of Psychology, University of Wales, Bangor LL57 2AS and

Walton Centre for Neurology and

Neurosurgery, Liverpool L9 7ป, UK

Correspondence to: R M Bracewell; m.bracewell@bangor.ac.uk

\section{REFERENCES}

1 Cauraugh JH , Kim SB. Stroke motor recovery: active neuromuscular stimulation and repetitive practice schedules. J Neurol Neurosurg Psychiatry 2003:74:1562-6.

2 Connor B, Harvey DA, Bracewell RM, et al. Response guided learning: research in cognitive rehabilitation following stroke. In: Sassoon R, eds. Understanding stroke. Lingfield: Pardoe Blacker, 2002.

3 Hallett $M$. Plasticity of the human motor cortex and recovery from stroke. Brain Res Rev 2001:36:169-74.

4 Ward NS, Brown MM, Thompson AJ, et al. Neural correlates of outcome after stroke: a crosssectional fMRI study. Brain 2003;126:1430-48.

5 Nudo RJ, Wise BM, SiFuentes F, et al. Neural substrates for the effects of rehabilitative training on motor recovery after ischaemic infarct. Science 1996:272:1791-4. 